Federal Reserve Bank of Minneapolis Research Department

\title{
Optimal Indirect and Capital Taxation
}

\author{
Mikhail Golosov, Narayana Kocherlakota, \\ and Aleh Tsyvinski*
}

Working Paper 615

June 2001

\begin{abstract}
In this paper, we consider an environment in which agents' productivities are private information, potentially multi-dimensional, and follow arbitrary stochastic processes. We allow for arbitrary incentive-compatible and physically feasible tax schemes. We prove that it is typically Pareto optimal to have positive capital taxes. As well, we prove that in any given period, it is Pareto optimal to tax consumption goods at a uniform rate.
\end{abstract}

*All, University of Minnesota and the Federal Reserve Bank of Minneapolis. Kocherlakota acknowledges the support of NSF SES-0076315. For comments and questions, email nkocher@econ.umn.edu. Versions of this paper were presented at New York University, UCLA, and UCSD; we thank the seminar participants for their comments. We thank Marco Bassetto, Larry Jones, Patrick Kehoe, Chris Phelan, Rui Zhao, and especially V. V. Chari for their comments. The views expressed herein are those of the authors and not necessarily those of the Federal Reserve Bank of Minneapolis or the Federal Reserve System. 


\section{Introduction}

Modern economic theory takes two different approaches to the problem of optimal taxation. One approach emphasizes the effects of taxation on capital accumulation (see Chari and Kehoe (1999) for an excellent survey). The basic assumption is that a government faces a dynamic Ramsey problem: it needs to fund a stream of purchases over time using linear taxes on capital and labor income. The hallmark result of this literature is that it is optimal for the government to set capital income tax rates to zero in the long run (Chamley (1986), Judd (1985)).

The second approach is based on the work of Mirrlees $(1971,1976)$ and abstracts entirely from capital accumulation. Instead, it models agents as being characterized by fixed heterogeneous and unobservable skill levels. The goal of taxation in this setting is to transfer resources from the highly skilled to the less skilled in an efficient way, given that incomes but not skills are observable. An important lesson of this literature is the uniform commodity taxation theorem of Atkinson and Stiglitz $(1976,1980)$. It states that if utility is weakly separable between consumption and leisure, then, despite the presence of the incentive problem, it is socially optimal for all consumption goods to be taxed at the same rate.

In this paper, we re-examine the zero capital income taxation and uniform commodity taxation theorems in a much more general setting than has been previously studied. We consider a dynamic version of Mirrlees' (1971) original model economy. In each period, multiple perishable consumption goods are produced using a number of different types of labor and an accumulable capital input. The agents have differential skills (productivities) at the different types of labor, and skills are heterogeneous over agents. Moreover, skills evolve stochastically over time in an arbitrary fashion (although this stochastic evolution is 
independent across agents). A crucial element of our approach is that we do not impose linearity (a la Ramsey) or piecewise differentiability (a la Mirrlees). We accomplish this generality by allowing for any incentive-compatible and physically feasible allocation.

This environment is technically challenging: it features both dynamically evolving private information, and a multiple-dimensional type space. There is no known way to develop a full characterization of the socially optimal allocations in this environment. In particular, simply plugging in first order conditions for the large number of incentive constraints might well lead to misleading conclusions.

In the first part of the paper, we re-consider the zero capital income taxation theorem. We specialize the environment to have only one consumption good. We assume also that utility is additively separable in consumption and leisure. We prove that in a Pareto optimal ${ }^{1}$ allocation, individual consumption satisfies a "reciprocal" intertemporal first order condition of the kind derived by Rogerson (1985):

$$
1 / u^{\prime}\left(c_{t}\right)=\left(\beta R_{t+1}\right)^{-1} E_{t}\left\{1 / u^{\prime}\left(c_{t+1}\right)\right\}
$$

Here, $R_{t+1}$ is the marginal return to investment, $u$ is the agent's momentary utility function, and $\beta$ is the individual discount factor.

This "reciprocal first order condition" has an important consequence. If individual consumption is not deterministic in a Pareto optimum, then from Jensen's inequality we

\footnotetext{
${ }^{1}$ By Pareto optimal, we mean Pareto optimal relative to the set of all allocations that are both incentivecompatible and physically feasible.
} 
know that for some $t$ :

$$
u^{\prime}\left(c_{t}\right)<\beta R_{t+1} E_{t} u^{\prime}\left(c_{t+1}\right)
$$

(The incentive problem means that it is typically efficient for individual consumption to be stochastic: The planner needs to offer more consumption to high productivity types to get them to work more.) We prove that (1) implies that if agents trade capital and consumption in a sequence of competitive markets, it is optimal for tax rates on capital income to be positive.

The intuition behind the inequality (1) (and the associated capital income tax result) is as follows. Suppose society considers increasing investment by lowering an individual's period $t$ consumption by $\varepsilon$ and raising an individual's period $(t+1)$ consumption by $\varepsilon R_{t+1}$. Doing so has two immediate consequences on social welfare (measured in utiles): there is a $\operatorname{cost} u^{\prime}\left(c_{t}\right) \varepsilon$ and a benefit $\beta \varepsilon R_{t+1} E_{t} u^{\prime}\left(c_{t+1}\right)$.

However, there is a third, less obvious, consequence for social welfare. By increasing an individual's consumption in period $(t+1)$ by $\varepsilon R_{t+1}$, society reduces the correlation between his period $(t+1)$ consumption and his period $(t+1)$ productivity. The purpose of this correlation is to provide incentives for the individual. Hence, the correlation reduction caused by the $\varepsilon$ increase in investment implies that the individual's period $(t+1)$ effort and period $(t+1)$ output falls.

This adverse effect on future incentives is an additional marginal social cost of increasing investment by $\varepsilon$. In a Pareto optimum, the sum of all marginal social costs is equated to the sum of all marginal social benefits. Hence, we obtain (1): the (partial) marginal cost $u^{\prime}\left(c_{t}\right)$ of additional investment is lower than the (total) marginal benefit $\beta R_{t+1} E_{t} u^{\prime}\left(c_{t+1}\right)$. 
We go on to re-consider the uniform commodity taxation theorem. We revert to the general assumption of multiple consumption goods, and assume that utility is weakly separable between consumption and labor. We prove that any Pareto optimal allocation has the property that within a period, the marginal rate of substitution between any two consumption goods, for any agent, equals the marginal rate of transformation between those goods. This result implies that if agents can trade consumption goods in a spot market, all consumption goods should be taxed uniformly.

The idea behind the proof of the uniform commodity taxation theorem is as follows. Because utility is weakly separable, consumption only affects the incentive constraints and the planner's objective function through the amount of utility derived from consumption. Hence, as long as resources are scarce, the planner wants to find a way to deliver these utilities that minimizes the resource cost of doing so. This immediately implies the uniform commodity taxation theorem.

We make two distinct contributions to public finance. The first contribution is that we find a general role for positive capital income taxes in a Pareto optimum. ${ }^{2}$ Here, we find that thinking based only on representative agent models can be misleading. It is the dynamic evolution of idiosyncratic shocks that makes positive capital income taxes optimal.

The second is that we greatly generalize the applicability of the uniform commodity taxation theorem. The standard proof of this result is based on much stronger assumptions.

\footnotetext{
${ }^{2}$ Aiyagari (1995) argues that positive capital income taxes are optimal in an incomplete markets setting. However, he considers only steady-states, rules out markets in an ad hoc basis, and allows only for linear taxes. In contrast, we consider all possible allocations that are feasible and incentive-compatible in a given environment, and thus allow for all possible taxation schemes.

Garriga (2001) shows that in overlapping generations contexts, the optimal linear tax on capital income may be non-zero.
} 
Atkinson and Stiglitz (1976)'s argument is made in a setting without capital or informational evolution. Moreover, the argument is made under restrictive assumptions: optimal taxes are differentiable and that a first-order approach is valid. Both assumptions are typically satisfied only under highly restrictive conditions. We simplify the proof and thereby greatly broaden the range of environments to which it applies.

It is important to re-emphasize that we prove our results while allowing individual productivities to follow arbitrary stochastic processes. There are still many significant unresolved empirical disputes about the time series structure of individual wages. (See Storesletten, Telmer and Yaron (2001) for a brief discussion of the ongoing controversy.) For this reason, we view the generality of our analysis as its most important feature.

\section{Setup}

The economy lasts for $T$ periods, where $T$ may be infinity, and has a unit measure of agents. The economy is endowed with $K_{1}^{*}$ units of the single capital good. There are $J$ consumption goods, which are produced by capital and labor at $N$ different tasks. The agents have identical preferences. The preferences of a given agent are von Neumann-Morgenstern, with cardinal utility function:

$$
\sum_{t=1}^{T} \beta^{t-1} U\left(c_{t}, l_{t}\right), 1>\beta>0
$$

where $c_{t} \in R_{+}^{J}$ is the agent's consumption in period $t$, and $l \in R_{+}^{N}$ is the amount of time spent working in period $t$ by the agent at the $N$ different tasks. We assume that $U$ is bounded from above or bounded from below; this guarantees that the utility from any consumption/labor 
process is well-defined as an element of the extended reals.

The agents' skills at the $N$ different tasks differ across agents and over time. We model this cross-sectional and temporal heterogeneity as follows. Let $\Theta$ be a Borel set in $R_{+}^{N}$, and let $\mu$ be a probability measure over the Borel sets that are subsets of $\Theta^{T}$. At the beginning of time, an element $\theta^{T}$ of $\Theta^{T}$ is drawn for each agent according to the measure $\mu$; the draws are independent across agents. This random vector $\theta^{T}$ is the agent's type; its $t$-th component $\theta_{t}$ is the agent's skill vector in period $t$. We assume that a law of large numbers applies: the measure of agents in the population with type $\theta^{T}$ in Borel set $B$ is given by $\mu(B)$.

What makes the information problem dynamic is that a given agent privately learns his $\theta_{t}$ at the beginning of period $t$ and not before. Thus, at the beginning of period $t$, an agent knows his history $\theta^{t}$ of current and past skill vectors but not his future skill vectors. We represent this information structure formally as follows. Define $P_{t}: \Theta^{T} \rightarrow \Theta^{t}$ to be the projection operator: $P_{t}\left(\theta_{1}, \ldots, \theta_{T}\right)=\left(\theta_{1}, \ldots, \theta_{t}\right)$. Then, define a $\sigma$-algebra $F_{t}=\left\{P_{t}^{-1}(B) \mid B \subset\right.$ $\Theta^{T}$ is Borel\}. An agent's information evolution can then be represented by the sequence $\left(F_{1}, F_{2}, \ldots, F_{T}\right)$ of $\sigma$-algebras.

Notice that this stochastic specification allows for virtually arbitrary dynamic evolution of an agent's skills. For example, the agent's skills could be constant over time (which is the traditional public finance assumption). Alternatively, the skills could follow stationary or nonstationary stochastic processes over time. The only real restriction is that the skill processes are independent across agents.

What is the economic impact of these skill vectors? An agent with type $\theta_{t}$ produces 
effective labor $y_{n t}$ in task $n$ according to the function:

$$
y_{n t}=\theta_{n t} l_{n t}
$$

where $l_{n t}$ is the amount of time spent working at task $n$. Effective labor $y_{n t}$ is observable, but actual labor $l_{n t}$ is not.

Along with the consumption goods, there is an accumulable capital good. We define an allocation in this society to be $(c, y, K)=\left(c_{t}, y_{t}, K_{t+1}\right)_{t=1}^{T}$ where for all $t$ :

$$
\begin{aligned}
& K_{t+1} \in R_{+} \\
& c_{t}: \Theta^{T} \rightarrow R_{+}^{J} \\
& y_{t}: \Theta^{T} \rightarrow R_{+}^{N} \\
& \left(c_{t}, y_{t}\right) \text { is } F_{t} \text {-measurable }
\end{aligned}
$$

Here, $y_{n t}\left(\theta^{T}\right)$ is the amount of effective labor at task $n$ produced by a type $\theta^{T}$ in period $t$, $c_{j t}\left(\theta^{T}\right)$ is the amount of the $j$ th consumption good given to a type $\theta^{T}$ in period $t$, and $K_{t+1}$ is the amount of capital carried over period $t$ into period $(t+1)$.

Let $G: R_{+}^{J+2+N} \rightarrow R$ be strictly increasing and continuously differentiable with respect to its first $(J+1)$ arguments, and strictly decreasing and continuously differentiable with respect to its $(J+2)$ th argument. This function tells us which vectors of capital input, labor inputs and consumption outputs are technologically available. Specifically, we assume that 
the initial endowment of capital is $K_{1}^{*}$, and define an allocation $(c, y, K)$ to be feasible if:

$$
\begin{aligned}
\left(\int c_{t} d \mu, \int y_{t} d \mu\right) & \in R_{+}^{J+N} \text { for all } t \\
G\left(\int c_{t} d \mu, K_{t+1}, K_{t}, \int y_{t} d \mu\right) & \leq 0 \text { for all } t \\
K_{1} & =K_{1}^{*}
\end{aligned}
$$

The first requirement is that $c_{t}$ and $y_{t}$ be integrable for all $t$.

Because $\theta^{T}$ is unobservable, allocations must respect incentive-compatibility conditions. A reporting strategy $\sigma$ is a mapping from $\Theta^{T}$ into $\Theta^{T}$ such that for all $t, \sigma_{t}$ is $F_{t^{-}}$ measurable. Let $\Sigma$ be the set of all possible reporting strategies, and define:

$$
\begin{aligned}
W(. ; c, y) & : \quad \Sigma \rightarrow R \\
W(\sigma ; c, y) & =\sum_{t=1}^{T} \beta^{t-1} \int U\left(c_{t}(\sigma),\left(y_{n t}(\sigma) / \theta_{n t}\right)_{n=1}^{N}\right) d \mu
\end{aligned}
$$

to be the utility from reporting strategy $\sigma$, given an allocation $(c, y)$. Let $\sigma^{*}$ be the truthtelling strategy $\left(\sigma^{*}\left(\theta^{T}\right)=\theta^{T}\right.$ for all $\left.\theta^{T}\right)$. Then, an allocation $(c, y, K)$ is incentive-compatible if:

$$
W\left(\sigma^{*} ; c, y\right) \geq W(\sigma ; c, y) \text { for all } \sigma \text { in } \Sigma
$$

An allocation which is incentive-compatible and feasible is said to be incentive-feasible. ${ }^{3}$

We allow for the possibility that the planner weights agents differently based on their

\footnotetext{
${ }^{3}$ We restrict attention to direct mechanisms. By the Revelation Principle, this is without loss of generality. As well, we restrict attention to mechanisms in which an individual's consumption and output depend only on his own announcements. This is without loss of generality because there is a continuum of agents with independent shock processes.
} 
initial skill levels. Specifically, let $\chi_{1}: \Theta^{T} \rightarrow R_{+}$be $F_{1}$-measurable, and suppose that $\int \chi_{1} d \mu=1$. Then, we define the following programming problem, $P 1\left(K_{1}\right)$, for an arbitrary level $K_{1}$ of initial capital:

$$
\begin{aligned}
\Gamma^{*}\left(K_{1}\right)= & \sup _{c, y, K} \sum_{t=1}^{T} \beta^{t-1} \int U\left(c_{t},\left(y_{n t} / \theta_{n t}\right)_{n=1}^{N}\right) \chi_{1} d \mu \\
& \text { s.t. } G\left(\int c_{t} d \mu, K_{t+1}, K_{t}, \int y_{t} d \mu\right) \leq 0 \text { for all } t \\
& W\left(\sigma^{*} ; c, y\right) \geq W(\sigma ; c, y) \text { for all } \sigma \text { in } \Sigma \\
& K_{1} \text { given } \\
& c_{t} \geq 0, y_{t} \geq 0, K_{t} \geq 0 \text { for all } t \text { and almost all } \theta^{T}
\end{aligned}
$$

We say that $\left(c^{*}, y^{*}, K^{*}\right)$ solves $P 1\left(K_{1}\right)$ if $\left(c^{*}, y^{*}, K^{*}\right)$ lies in the constraint set of $P 1\left(K_{1}\right)$ and:

$$
\Gamma^{*}\left(K_{1}\right)=\sum_{t=1}^{T} \beta^{t-1} \int U\left(c_{t}^{*},\left(y_{n t}^{*} / \theta_{n t}\right)_{n=1}^{N}\right) \chi_{1} d \mu
$$

In the actual model economy, there are initially $K_{1}^{*}$ units of capital. Hence, the planner's problem is to solve $P 1\left(K_{1}^{*}\right)$. We assume throughout that there is a solution to $P 1\left(K_{1}^{*}\right)$ and that $\left|\Gamma^{*}\left(K_{1}^{*}\right)\right|<\infty$. Any solution to $P 1\left(K_{1}^{*}\right)$ is a Pareto optimum. ${ }^{4}$

Note that the planner's maximized objective $\Gamma^{*}$ is weakly increasing. In our analysis, we will often require that $\Gamma^{*}$ is strictly increasing. The following lemma shows that, under a mild regularity condition, $\Gamma^{*}$ is strictly increasing if $U$ is additively separable between consumption and leisure. (In the remainder of the paper, as is standard, we use the terms

\footnotetext{
${ }^{4}$ Specifically, any solution to $P 1\left(K_{1}^{*}\right)$ is interim Pareto optimal, conditional on the realization of $\theta_{1}$. If $\chi=1$, the solutions to $P 1\left(K_{1}^{*}\right)$ are symmetric ex-ante Pareto optima.
} 
for almost all $\theta^{T}$ and almost everywhere (or a.e.) equivalently.)

Lemma 1 . Let $U(c, l)=u(c)-v(l)$, where $u$ is strictly increasing and continuously differentiable. Suppose that for any $\left(c^{*}, y^{*}, K^{*}\right)$ that solves $P 1\left(K_{1}^{*}\right)$, there exists some $t$ and positive scalars $c^{+}, c_{+}$such that $c^{+} \geq c_{j t}^{*} \geq c_{+}$a.e. for all $j$. Then, $\Gamma^{*}\left(K_{1}\right)<\Gamma^{*}\left(K_{1}^{*}\right)$ for all $K_{1}<K_{1}^{*}$.

Proof. Suppose $\Gamma^{*}\left(K_{1}\right)=\Gamma^{*}\left(K_{1}^{*}\right)$ for some $K_{1}<K_{1}^{*}$. Let $\left(c^{*}, y^{*}, K^{*}\right)$ solve $P 1\left(K_{1}\right)$ and also $P 1\left(K_{1}^{*}\right)$. Without loss of generality, assume that $c_{1}^{*}$ satisfies the uniform boundedness conditions. Define $c_{11}^{\prime}\left(\theta^{T}, \varepsilon\right)$ to be the solution to the equation:

$$
u\left(c_{11}^{\prime}\left(\theta^{T}, \varepsilon\right),\left(c_{1 j}^{*}\left(\theta^{T}\right)\right)_{j \neq 1}\right)-u\left(c_{1}^{*}\left(\theta^{T}\right)\right)=\varepsilon \text { for all } \theta^{T}
$$

for $\varepsilon$ nonnegative. Here, $c_{11}^{\prime}\left(\theta^{T}, \varepsilon\right)$ is the amount of consumption good 1 that gives a type $\theta^{T} \varepsilon$ more utiles than $c_{1}^{*}$. Clearly, $c_{11}^{\prime}$ is $F_{1}$-measurable with respect to $\theta^{T}$, and is continuous with respect to $\varepsilon$.

From the mean value theorem, for $\varepsilon$ small, we know that:

$$
\left|c_{11}^{\prime}\left(\theta^{T}, \varepsilon\right)-c_{11}^{*}\left(\theta^{T}\right)\right|=\varepsilon / u_{1}\left(c_{11}^{\prime}\left(\theta^{T}, \varepsilon^{\prime}\right),\left(c_{1 j}^{*}\left(\theta^{T}\right)\right)_{j \neq 1}\right), 0<\varepsilon^{\prime}<\varepsilon
$$

where $u_{1}$ is the partial of $u$ with respect to its first argument. From the regularity conditions on $c^{*}$, we know that there exists $M>0$ such that:

$$
\left|c_{11}^{\prime}\left(\theta^{T}, \varepsilon\right)-c_{11}^{*}\left(\theta^{T}\right)\right|<M \varepsilon \text { for } \varepsilon \text { small }
$$

Hence, for $\varepsilon$ small, $c_{11}^{\prime}\left(\theta^{T}, \varepsilon\right)$ is integrable as a function of $\theta^{T}$. Moreover, adding $\varepsilon$ to initial 
consumption is feasible for initial capital $K_{1}^{*}$, as long as $\varepsilon$ is sufficiently small. That is, for sufficiently small $\varepsilon$,

$$
G\left(\int c_{1}^{\prime}\left(\theta^{T}, \varepsilon\right) d \mu, K_{2}^{*}, K_{1}^{*}, \int y^{*} d \mu\right)<0
$$

where $c_{1}^{\prime}\left(\theta^{T}, \varepsilon\right) \equiv\left(c_{11}^{\prime}\left(\theta^{T}, \varepsilon\right),\left(c_{1 j}^{*}\left(\theta^{T}\right)\right)_{j \neq 1}\right)$. Thus, $\left(c^{\prime}, y^{*}, K^{*}\right)$ is feasible, given initial capital $K_{1}^{*}$

For all $\theta^{T}$,

$$
\begin{aligned}
& u\left(c_{1}^{\prime}\left(\theta^{T}, \varepsilon\right)\right)-v\left(\left(y_{n 1}^{*}\left(\theta^{T}\right) / \theta_{n 1}\right)_{n=1}^{N}\right) \\
= & u\left(c_{1}^{*}\left(\theta^{T}\right)\right)+\varepsilon-v\left(\left(y_{n 1}^{*}\left(\theta^{T}\right) / \theta_{n 1}\right)_{n=1}^{N}\right) \\
\geq & u\left(c_{1}^{*}\left(\theta^{T \prime}\right)\right)+\varepsilon-v\left(\left(y_{n 1}^{*}\left(\theta^{T \prime}\right) / \theta_{n 1}\right)_{n=1}^{N}\right) \\
= & u\left(c_{1}^{\prime}\left(\theta^{T \prime}, \varepsilon\right)\right)-v\left(\left(y_{n 1}^{*}\left(\theta^{T \prime}\right) / \theta_{n 1}\right)_{n=1}^{N}\right)
\end{aligned}
$$

which proves that $\left(c^{\prime}, y^{*}\right)$ is incentive-compatible. It follows that $\left(c^{*}, y^{*}\right)$ cannot be a solution to $P 1\left(K_{1}^{*}\right)$.

When utility is additively separable, there is a simple incentive-compatible way to spread extra resources across the various types.

\section{Capital Income Taxes}

To obtain results about the intertemporal characteristics of optimal taxation, we simplify the model. We set the number of consumption goods $J=1$, and set:

$$
G\left(C_{t}, Y_{t}, K_{t}, K_{t+1}\right)=C_{t}+K_{t+1}-K_{t}(1-\delta)-\Phi\left(K_{t}, Y_{t}\right)
$$


where $\Phi$ is strictly increasing and continuously differentiable in its first argument. (These restrictions on $J$ and $G$ do not apply in the next section.) Throughout the section, we assume that the partial derivative $U_{c}$ exists and is continuous in its first argument over the positive reals. We proceed by first providing a partial characterization of Pareto optima, and then establishing the implications of this characterization for capital income tax rates.

\section{A. Characterizing Pareto Optima}

The main result in this section is similar to (but much more general than) that derived by Rogerson (1985) for optimal contracts in relationships with repeated moral hazard. We obtain a restriction on the intertemporal behavior of consumption by minimizing the amount of initial capital necessary to deliver a given amount of utility to each possible type of agent. We use the notation $E\left\{. \mid F_{t}\right\}$ to denote the conditional expectation operator.

Theorem 1. Let $U(c, l)=u(c)-v(l)$. Suppose $\left(c^{*}, y^{*}, K^{*}\right)$ solves $P 1\left(K_{1}^{*}\right)$, and that there exists $t<T$ and scalars $M^{+}, M_{+}$such that $M^{+} \geq c_{t}^{*}, c_{t+1}^{*}, K_{t+1}^{*} \geq M_{+}>0$ a.e.. Then:

$$
\beta\left(1-\delta+\Phi_{K}\left(K_{t+1}^{*}, \int y_{t+1}^{*} d \mu\right)\right)=E\left\{u^{\prime}\left(c_{t}^{*}\left(\theta^{t}\right) / u^{\prime}\left(c_{t+1}^{*}\left(\theta^{t+1}\right)\right) \mid F_{t}\right\}\right.
$$

Proof. Define:

$$
L^{\infty}\left(F_{t}\right)=\left\{x F_{t^{-}} \text {measurable } \mid \exists A \in F_{t} \text { s.t. } \mu(A)=1 \text { and } \sup _{\theta^{T} \in A}|x|<\infty\right\}
$$

Consider the following minimization problem $M I N 1$ :

$$
\min _{\eta_{t}, \varepsilon_{t+1}, \zeta_{t}}\left[\zeta_{t}+\int \eta_{t} d \mu\right]
$$




$$
\begin{aligned}
& \text { s.t. } \\
& \int \varepsilon_{t+1} d \mu=\Phi\left(K_{t+1}^{*}+\zeta_{t}, \int y_{t+1}^{*} d \mu\right)-\Phi\left(K_{t+1}^{*}, \int y_{t+1}^{*} d \mu\right)+(1-\delta) \zeta_{t} \\
& u\left(c_{t}^{*}+\eta_{t}\right)+\beta u\left(c_{t+1}^{*}+\varepsilon_{t+1}\right)=u\left(c_{t}^{*}\right)+\beta u\left(c_{t+1}^{*}\right) \text { a.e. } \\
& c_{t}^{*}+\eta_{t} \geq 0, c_{t+1}^{*}+\varepsilon_{t+1} \geq 0, K_{t+1}^{*}+\zeta_{t} \geq 0 \text { a.e. } \\
& \eta_{t} \in L^{\infty}\left(F_{t}\right), \varepsilon_{t+1} \in L^{\infty}\left(F_{t+1}\right), \zeta_{t} \in R
\end{aligned}
$$

We claim that $M I N 1$ is solved by setting $\left(\eta_{t}, \varepsilon_{t+1}, \zeta_{t}\right)=0$. Suppose not, and that there exists some element $\left(\eta_{t}, \varepsilon_{t+1}, \zeta_{t}\right)$ of the constraint set which generates a negative value for the objective. There exists a subset $B$ of $\Theta^{T}$ such that $\mu(B)=1$ and:

$$
\begin{aligned}
& u\left(c_{t}^{*}\left(\theta^{T}\right)+\eta_{t}\left(\theta^{T}\right)\right)+\beta u\left(c_{t+1}^{*}\left(\theta^{T}\right)+\varepsilon_{t+1}\left(\theta^{T}\right)\right) \\
= & u\left(c_{t}^{*}\left(\theta^{T}\right)\right)+\beta u\left(c_{t+1}^{*}\left(\theta^{T}\right)\right) \text { for all } \theta^{T} \text { in } B
\end{aligned}
$$

Define $\left(c^{\prime}, K^{\prime}\right)$ so that $c^{\prime}=c^{*}$ and $K^{\prime}=K^{*}$ except that:

$$
\begin{aligned}
c_{t}^{\prime}\left(\theta^{T}\right) & =c_{t}^{*}\left(\theta^{T}\right)+\eta_{t}\left(\theta^{T}\right) \text { for all } \theta^{T} \text { in } B \\
c_{t+1}^{\prime}\left(\theta^{T}\right) & =c_{t+1}^{*}\left(\theta^{T}\right)+\varepsilon_{t+1}\left(\theta^{T}\right) \text { for all } \theta^{T} \text { in } B \\
K_{t+1}^{\prime} & =K_{t+1}^{*}+\zeta_{t}
\end{aligned}
$$

We claim that $\left(c^{\prime}, y^{*}, K^{\prime}\right)$ is incentive-feasible, delivers the same value of the planner's objective as $\left(c^{*}, y^{*}, K^{*}\right)$ and uses fewer resources. The allocation $\left(c^{\prime}, y^{*}, K^{\prime}\right)$ is obviously 
feasible because:

$$
\begin{aligned}
\int c_{t}^{\prime} d \mu+K_{t+1}^{\prime} & =\int c_{t}^{*} d \mu+K_{t+1}^{*}+\zeta_{t}+\int \eta_{t} d \mu \\
& <\int c_{t}^{*} d \mu+K_{t+1}^{*}
\end{aligned}
$$

We next want to show that the allocation $\left(c^{\prime}, y^{*}, K^{\prime}\right)$ is incentive-compatible. By construction:

$$
\begin{aligned}
& u\left(c_{t}^{\prime}\left(\theta^{T}\right)\right)+\beta u\left(c_{t+1}^{\prime}\left(\theta^{T}\right)\right) \\
= & u\left(c_{t}^{*}\left(\theta^{T}\right)\right)+\beta u\left(c_{t+1}^{*}\left(\theta^{T}\right)\right) \text { for all } \theta^{T}
\end{aligned}
$$

(not just $\theta^{T}$ in $B$ ). Then, we know that for any $\sigma$ in $\Sigma$ and for all $\theta^{T}$ :

$$
\begin{aligned}
& \sum_{s=1}^{T} \beta^{s-1} u\left(c_{s}^{\prime}\left(\sigma\left(\theta^{T}\right)\right)\right) \\
= & \sum_{s=1}^{t-1} \beta^{s-1} u\left(c_{s}^{*}\left(\sigma\left(\theta^{T}\right)\right)\right)+\beta^{t-1}\left[u\left(c_{t}^{\prime}\left(\sigma\left(\theta^{T}\right)\right)\right)+\beta u\left(c_{t+1}^{\prime}\left(\sigma\left(\theta^{T}\right)\right)\right)\right]+\sum_{s=t+2}^{T} \beta^{s-1} u\left(c_{s}^{*}\left(\sigma\left(\theta^{T}\right)\right)\right) \\
= & \sum_{s=1}^{t-1} \beta^{s-1} u\left(c_{s}^{*}(\sigma)\right)+\beta^{t-1}\left[u\left(c_{t}^{*}\left(\sigma\left(\theta^{T}\right)\right)\right)+\beta u\left(c_{t+1}^{*}\left(\sigma\left(\theta^{T}\right)\right)\right)\right]+\sum_{s=t+2}^{T} \beta^{s-1} u\left(c_{s}^{*}\left(\sigma\left(\theta^{T}\right)\right)\right) \\
= & \sum_{s=1}^{T} \beta^{s-1} u\left(c_{s}^{*}\left(\sigma\left(\theta^{T}\right)\right)\right)
\end{aligned}
$$

This means that for any $\sigma$, agents get the same utility from $c^{\prime}$ as from $c^{*}$. It follows that $\left(c^{\prime}, y^{*}\right)$ is incentive-compatible:

$$
\begin{aligned}
& \int \sum_{t=1}^{T} \beta^{t-1}\left[u\left(c_{t}^{\prime}\right)-v\left(\left(y_{n t}^{*} / \theta_{n t}\right)_{n=1}^{N}\right)\right] d \mu \\
= & \int \sum_{t=1}^{T} \beta^{t-1}\left[u\left(c_{t}^{*}\right)-v\left(\left(y_{n t}^{*} / \theta_{n t}\right)_{n=1}^{N}\right)\right] d \mu
\end{aligned}
$$




$$
\begin{aligned}
& \geq \int \sum_{t=1}^{T} \beta^{t-1}\left[u\left(c_{t}^{*}(\sigma)\right)-v\left(\left(y_{n t}^{*}(\sigma) / \theta_{n t}\right)_{n=1}^{N}\right)\right] d \mu \text { for any } \sigma \\
& =\int \sum_{t=1}^{T} \beta^{t-1}\left[u\left(c_{t}^{\prime}(\sigma)\right)-v\left(\left(y_{n t}^{*}(\sigma) / \theta_{n t}\right)_{n=1}^{N}\right)\right] d \mu
\end{aligned}
$$

The inequality comes from the fact that $\left(c^{*}, y^{*}\right)$ is incentive-compatible.

Hence, $\left(c^{\prime}, y^{*}, K^{\prime}\right)$ uses fewer resources, is incentive-compatible, and delivers the same value of the objective to the planner. This violates Lemma 1.

We can therefore characterize $\left(c^{*}, K^{*}\right)$ using the first order conditions of $M I N 1$. Here, we do this for the case in which $\Theta$ is finite. We establish the first order conditions more generally in the appendix.

Suppose $\theta^{t}$ has positive probability under $\mu$, and let $\mu\left\{\theta^{t}, \theta_{t+1}\right\}$ denote the probability of $\left(\theta^{t}, \theta_{t+1}\right)$. Let $\lambda_{t+1}$ be the multiplier on the resource constraint, and let $\nu\left(\theta_{t+1}\right) \mu\left\{\theta^{t}, \theta_{t+1}\right\}$ be the multiplier on the utility constraint for state $\theta_{t+1}$. The first order conditions of $M I N 1$ with respect to $(\eta, \zeta, \varepsilon)$ are:

$$
\begin{aligned}
& \mu\left\{\theta_{t}\right\}-u^{\prime}\left(c_{t}^{*}\left(\theta^{t}\right)\right) \sum_{\theta_{t+1} \in \Omega_{t}\left(\theta^{t}\right)} \nu\left(\theta_{t+1}\right) \mu\left\{\theta^{t}, \theta_{t+1}\right\}=0 \\
& \lambda_{t+1}\left[(1-\delta)+\Phi_{K}\left(K_{t+1}^{*}, \int y_{t+1}^{*} d \mu\right)\right]=1 \\
& \lambda_{t+1}-\beta u^{\prime}\left(c_{t+1}^{*}\left(\theta^{t+1}\right)\right) \nu\left(\theta_{t+1}\right)=0 \text { for all } \theta_{t+1} \in \Omega_{t}\left(\theta^{t}\right)
\end{aligned}
$$

where $\Omega_{t}\left(\theta^{t}\right)$ is the set of $\theta_{t+1}$ that have positive probability conditional on $\theta^{t}$. By substituting out for $\nu$, we get:

$$
u^{\prime}\left(c_{t}^{*}\left(\theta^{t}\right)\right) E\left\{1 / u^{\prime}\left(c_{t+1}^{*}\left(\theta^{t+1}\right)\right) \mid \theta^{t}\right\}=\beta / \lambda_{t+1}
$$

By substituting out for $\lambda_{t+1}$, we get the theorem. 
The intertemporal restriction stated in Theorem 1 may seem unintuitive. We can connect it back to the usual first-order condition for individual capital accumulation when agents are uncertain about their future consumption. This standard first-order condition has the form:

$$
E_{t} z_{t+1}=1
$$

where $z_{t}=\beta\left(1-\delta+\Phi_{K}\left(K_{t}, \int y_{t} d \mu\right)\right) u^{\prime}\left(c_{t}\right) / u^{\prime}\left(c_{t-1}\right)$. The theorem states that because of the informational frictions, the optimum obeys the following first-order condition instead:

$$
E_{t}\left(1 / z_{t+1}\right)=1
$$

Clearly, if $z_{t+1}$ is perfectly predictable using period $t$ information, then these first-order conditions are equivalent. Otherwise, they differ.

This kind of thinking informs the next two corollaries. The first concerns the (typical) case in which $c_{t}^{*}$ is not perfectly predictable.

Corollary 1. Let $U(c, l)=u(c)-v(l)$. Suppose $\left(c^{*}, y^{*}, K^{*}\right)$ solves $P 1\left(K_{1}^{*}\right)$, and that there exists $t<T$ and scalars $M^{+}, M_{+}$such that $M^{+} \geq c_{t}^{*}, c_{t+1}^{*}, K_{t+1}^{*} \geq M_{+}>0$ a.e.. Suppose also that $\int\left[\operatorname{Var}\left(c_{t+1}^{*} \mid F_{t}\right)\right] d \mu>0$. Then with positive probability:

$$
u^{\prime}\left(c_{t}^{*}\right)<\beta\left(1-\delta+\Phi_{K}\left(K_{t+1}^{*}, \int y_{t+1}^{*} d \mu\right)\right) E\left\{u^{\prime}\left(c_{t+1}^{*}\left(\theta^{t+1}\right)\right) \mid F_{t}\right\}
$$

Proof. Simply apply Jensen's inequality to the condition in Theorem 1. 
This corollary says that if $c_{t+1}^{*}$ is not predictable given $F_{t}$, the expected marginal utility of investing in capital is higher than the marginal utility of current consumption. Note that this lack of predictability is to be expected in general because the planner wants to elicit high labor from high productivity types.

It is interesting to contrast Corollary 1 with the results concerning optimal linear taxation of capital and labor income in a representative agent economy. Chamley (1986) and Judd (1985) prove for a general specification of $u$ that it is optimal in the long run to eliminate the wedge between expected marginal utility of investing in capital and the marginal utility of current consumption. Indeed, when $u(c)=c^{1-\sigma} /(1-\sigma)$, Chamley proves an even stronger result: it is optimal for the wedge to be zero for all $t$, not just in the long run. In contrast, we find that for any specification of $u$, as long as $c_{t+1}^{*}$ is not predictable given $F_{t}$, the tax on capital income in period $t$ should be positive.

There are special circumstances in which the inequality in Corollary 1 becomes an equality instead. In particular, if agents have fixed productivities over time, then the Pareto optimal allocations display no wedge between the marginal utility of consumption and the expected marginal utility of investment.

Corollary 2. Suppose that $\mu(B)>0$ only if $\mu(B)=\mu\left\{\theta^{T} \in B \mid \theta_{t}=\theta_{1}\right.$ for all $\left.t\right\}$. Let $U(c, l)=u(c)-v(l)$. Suppose $\left(c^{*}, y^{*}, K^{*}\right)$ solves $P 1\left(K_{1}^{*}\right)$, and that there exists $t<T$ and scalars $M^{+}, M_{+}$such that $M^{+} \geq c_{t}^{*}, c_{t+1}^{*}, K_{t+1}^{*} \geq M_{+}>0$ a.e.. Then:

$$
\beta u^{\prime}\left(c_{t+1}^{*}\right)\left(1-\delta+\Phi_{K}\left(K_{t+1}^{*}, \int y_{t+1}^{*} d \mu\right)\right) / u^{\prime}\left(c_{t}^{*}\right)=1 \text { a.e. }
$$


This corollary follows from the fact that $\theta_{t}$ is perfectly predictable, given $\theta_{1}$. In fact, using a similar approach as in Theorem 1, we can prove (at least when $\Theta$ is finite) that even if preferences are non-separable between consumption and labor, we obtain a version of Chamley-Judd's classic result for this case of fixed productivities.

Proposition 1. Suppose $T=\infty, \Theta$ is finite, and that $\mu\left\{\theta^{\infty}\right\}>0$ iff $\theta_{t}=\theta_{1}$ for all $t$. Suppose that $\Gamma^{*}\left(K_{1}\right)<\Gamma^{*}\left(K_{1}^{*}\right)$ for all $K_{1}<K_{1}^{*}$. Let a strictly positive allocation $\left(c^{*}, y^{*}, K^{*}\right)$ solve $P 1\left(K_{1}^{*}\right)$, and suppose that for all $\theta_{1}$, the sequence $\left\{c_{t}^{*}\left(\theta_{1}\right), y_{t}^{*}\left(\theta_{1}\right), K_{t}^{*}\right\}_{t=1}^{\infty}$ converges to a positive limit $\left(c_{s s}\left(\theta_{1}\right), y_{s s}\left(\theta_{1}\right), K_{s s}\right)$. Then:

$$
\beta^{-1}=1+\Phi_{K}\left(K_{s s}, \int y_{s s} d \mu\right)-\delta
$$

Proof. We claim that $\left(c^{*}, K^{*}\right)$ solves the following minimization problem:

$$
\begin{aligned}
& \min _{c,\left(K_{t}\right)_{t=1}^{T+1}} K_{1} \\
& \text { s.t. } \int c_{t} d \mu+K_{t+1}=K_{t}(1-\delta)+\Phi\left(K_{t}, \int y_{t}^{*} d \mu\right) \text { for all } t \\
& \sum_{t=1}^{\infty} \beta^{t-1} U\left(c_{t}\left(\theta_{1}\right),\left(\frac{y_{n t}^{*}\left(\theta_{1}\right)}{\hat{\theta}_{n 1}}\right)_{n=1}^{N}\right)=\sum_{t=1}^{\infty} \beta^{t-1} U\left(c_{t}^{*}\left(\theta_{1}\right),\left(\frac{y_{n t}^{*}\left(\theta_{1}\right)}{\hat{\theta}_{n 1}}\right)_{n=1}^{N}\right) \text { for all } \theta_{1}, \hat{\theta}_{1} \\
& K_{t} \in R_{+}, c_{t} \geq 0 \text { for all } t
\end{aligned}
$$

Suppose not. Then, there exists nonnegative $\left(c^{\prime}, K^{\prime}\right)$ such that $K_{1}^{\prime}<K_{1}^{*}$ and:

$$
\begin{aligned}
\int c_{t}^{\prime} d \mu+K_{t+1}^{\prime} & =K_{t}^{\prime}(1-\delta)+\Phi\left(K_{t}^{\prime}, \int y_{t}^{*} d \mu\right) \text { for all } t \\
\sum_{t=1}^{\infty} \beta^{t-1} U\left(c_{t}^{\prime}\left(\theta_{1}\right),\left(\frac{y_{n t}^{*}\left(\theta_{1}\right)}{\hat{\theta}_{n 1}}\right)_{n=1}^{N}\right) & =\sum_{t=1}^{\infty} \beta^{t-1} U\left(c_{t}^{*}\left(\theta_{1}\right),\left(\frac{y_{n t}^{*}\left(\theta_{1}\right)}{\hat{\theta}_{n 1}}\right)_{n=1}^{N}\right) \text { for all } \theta_{1}, \hat{\theta}_{1}
\end{aligned}
$$


It is clear that $\left(c^{\prime}, y^{*}, K^{\prime}\right)$ is feasible; $\left(c^{\prime}, y^{*}\right)$ is incentive-compatible because we have kept the utility of all announcement/true type pairs the same. This allocation solves $P 1\left(K_{1}\right)$, for $K_{1}<K_{1}^{*}$, which violates the assumption that $\Gamma^{*}$ is strictly increasing.

Now, we can characterize $\left(c^{*}, y^{*}, K^{*}\right)$ using the first order conditions to this problem. Let $\lambda_{t}$ be the multiplier on the period $t$ feasibility constraint and let $\gamma\left(\theta_{1}, \hat{\theta}_{1}\right)$ be the multiplier on the appropriate utility constraint.

Abusing notation slightly, we use $\mu\left(\theta_{1}\right)$ to denote $\mu\left\{\left(\theta_{1}, \theta_{1}, \theta_{1}, \ldots\right)\right\}$. Differentiating with respect to $c_{t}\left(\theta_{1}\right)$ for any $\theta_{1}$, we obtain:

$$
\sum_{\hat{\theta}_{1}} \gamma\left(\theta_{1}, \hat{\theta}_{1}\right) \beta^{t-1} U_{c}\left(c_{t}^{*}\left(\theta_{1}\right),\left(\frac{y_{n t}^{*}\left(\theta_{1}\right)}{\hat{\theta}_{n 1}}\right)_{n=1}^{N}\right)=\lambda_{t} \mu\left(\theta_{1}\right)
$$

where $U_{c}$ is the partial derivative of $U$ with respect to $c$. Differentiating with respect to $K_{t+1}$ we obtain:

$$
\lambda_{t}=\lambda_{t+1}\left(1+\Phi_{K}\left(K_{t+1}, \int y_{t+1}^{*} d \mu\right)-\delta\right)
$$

The assumption that $\left(c_{t}\left(\theta_{1}\right), y_{t}\left(\theta_{1}\right), K_{t}\right)$ converges to a positive limit for all $\theta_{1}$ guarantees that:

$$
\begin{aligned}
& \lim _{t \rightarrow \infty} \lambda_{t} / \lambda_{t+1}=1 / \beta \\
& \lim _{t \rightarrow \infty} \lambda_{t} / \lambda_{t+1}=\left(1+\Phi_{K}\left(K_{t+1}, \int y_{t+1}^{*} d \mu\right)-\delta\right)
\end{aligned}
$$

This implies the proposition. 


\section{B. Capital Trading and Capital Income Taxes}

The above results concern the wedges (or lack thereof) between marginal rates of substitution and transformation in Pareto optima. We now allow agents to trade consumption and capital in a sequence of competitive markets. We prove that in this sequential markets setting, our previous results about wedges translate directly into conclusions about capital income taxes (as long as utility is additively separable). We assume throughout this subsection that $\Phi$ is strictly concave in its first argument, that $T$ is finite, and that $\Theta$ is finite. (We believe, though, that the results are robust to relaxing the latter two assumptions.)

It is useful to isolate our questions about optimal capital income taxes from the (considerably more difficult) ones about optimal labor income taxes. To do so, we consider a class of capital-trading mechanisms that work as follows. In each period, each agent makes a report from the set $\Theta$ to a social planner. Based on the history of these reports, each agent receives some amount of consumption as after-tax income and is told what vector of effective labor to provide.

Up until this point, the capital-trading mechanisms are standard direct mechanisms. The difference is that agents need not consume their income processes. Instead, they can exchange capital and consumption, and rent out capital services, in a sequence of competitive markets. In each period, an agent faces a linear tax on his capital rental income; the tax rate may be a function of his history of reports.

The other side of the capital rental market is assumed to be a single representative firm. The firm is also partially centralized, because it is simply endowed with a sequence of effective labor which it cannot alter. However, the firm can freely rent capital from the agents; firm profits are split evenly among the agents in the economy. 
Thus, under a capital-trading mechanism, labor and after-tax income are allocated according to a direct mechanism. However, agents are allowed to engage in decentralized trade in capital markets. The only restriction is that they face (possibly report-contingent) tax rates on their capital income.

Formally, a capital-trading mechanism is a specification $(z, y, \tau)=\left(z_{t}, y_{t}, \tau_{t}\right)_{t=1}^{T}$ such that:

$$
\begin{aligned}
& z_{t}: \Theta^{T} \rightarrow R_{+} \\
& y_{t}: \Theta^{T} \rightarrow R_{+}^{N} \\
& \tau_{t}: \Theta^{T} \rightarrow R \\
& \left(z_{t}, y_{t}, \tau_{t+1}\right) \text { is } F_{t} \text {-measurable }
\end{aligned}
$$

Here, we interpret $z$ as an after-tax income process, $y$ as an effective labor process, and $\tau$ as the tax rate on capital income. Thus, given $(z, y, \tau)$, and a rental rate sequence $r \in R_{+}^{T}$, a typical agent, initially endowed with $K_{1}^{*}$ units of capital, solves the problem:

$$
\begin{aligned}
& \max _{(c, k, \sigma)} \int \sum_{t=1}^{T} \beta^{t-1} U\left(c_{t},\left(y_{n t}(\sigma) / \theta_{n t}\right)_{n=1}^{N}\right) d \mu \\
& \text { s.t. } c_{t}+k_{t+1} \leq k_{t}\left(1+r_{t}\left(1-\tau_{t}(\sigma)\right)-\delta\right)+z_{t}(\sigma) \\
& c_{t} \geq 0, k_{t} \geq 0, k_{1}=K_{1}^{*} \\
& c_{t}, k_{t+1} F_{t} \text {-measurable } \\
& \sigma \in \Sigma
\end{aligned}
$$


Note that agents take into account their ability to trade in the sequential capital markets when they are making their reports about their types. Their after-tax incomes and their capital income tax rates depend on their reports.

There is a representative firm which operates every period. Given $y$, and a rental rate sequence $r$, the firm solves the following deterministic maximization problem:

$$
\max _{K_{t} \geq 0} \Phi\left(K_{t}, \int y_{t} d \mu\right)-r_{t} K_{t}
$$

in each period. We assume that firm profits are split evenly among the agents, and so are embedded directly into $z_{t}$.

Given a capital-trading mechanism $(z, y, \tau),(c, k, r, K)$ is a sequential markets equilibrium if it satisfies three conditions. First, $\left(c, k, \sigma^{*}\right)$ solves the agent's problem given $(z, y, \tau, r)$. (Recall that $\sigma^{*}$ is the truth-telling strategy in $\Sigma$.) Second, $K$ solves the firm's problem, given $(y, r)$. Finally, markets clear in every period:

$$
\begin{aligned}
\int c_{t} d \mu+K_{t+1} & =\Phi\left(K_{t}, \int y_{t} d \mu\right)+(1-\delta) K_{t} \\
\int k_{t} d \mu & =K_{t}
\end{aligned}
$$

We now prove two results about capital-trading mechanisms. Both require the assumption that utility is additively separable. The first result is that any incentive-feasible allocation is a sequential markets equilibrium of some capital-trading mechanism. The key to the result is that all agents, regardless of their type, have the same preferences over consumption processes. 
Proposition 2. Let $U(c, l)=u(c)-v(l)$, where $u^{\prime},-u^{\prime \prime}>0$. Suppose $\left(c^{*}, y^{*}, K^{*}\right)$ is incentive-feasible and $\left(c_{t}^{*}, K_{t+1}^{*}\right)>0$ for all t. Then, there exists $(k, r, z, \tau)$ such that $\left(c^{*}, k, r, K^{*}\right)$ is an equilibrium of a capital-trading mechanism $\left(z, y^{*}, \tau\right)$.

Proof. Given $\left(c^{*}, y^{*}, k^{*}\right)$, define:

$$
\begin{aligned}
& k_{t}=K_{t}^{*} \\
& r_{t}=\Phi_{K}\left(K_{t}^{*}, \int y_{t}^{*} d \mu\right) \\
& \tau_{t+1}=1-\left(-1+\delta+u^{\prime}\left(c_{t}^{*}\right) /\left[\beta E\left\{u^{\prime}\left(c_{t+1}^{*}\right) \mid F_{t}\right\}\right]\right) / r_{t+1} \\
& z_{t}=c_{t}^{*}+K_{t+1}^{*}-K_{t}^{*}\left(1+r_{t}\left(1-\tau_{t}\right)-\delta\right)
\end{aligned}
$$

$K$ is clearly optimal for the firm given the rental rate sequence $r$ and the (aggregate) effective labor sequence $\int y d \mu$. We need to show that $\left(c^{*}, k^{*}\right)$ solves the agent's problem given $\left(z, y^{*}, \tau, r\right)$.

To do so, fix any reporting strategy $\sigma$. Conditional on this strategy, the agent faces the decision problem:

$$
\begin{aligned}
& \max _{(c, k)} \sum_{t=1}^{T} \beta^{t-1} \int u\left(c_{t}\right) d \mu \\
& \text { s.t. } c_{t}+k_{t+1}=k_{t}\left(1+r_{t}\left(1-\tau_{t}(\sigma)\right)-\delta\right)+z_{t}(\sigma) \\
& c_{t}, k_{t+1} F_{t} \text {-measurable } \\
& c_{t} \geq 0, k_{t} \geq 0, k_{1}=K_{1}^{*}
\end{aligned}
$$

We claim that the solution to this problem is to set $k_{t}=K_{t}^{*}$ and $c_{t}=c_{t}^{*}$. The choice set is 
convex. Clearly, these choices satisfy the agent's intertemporal first order conditions. They also satisfy his flow budget constraints because of the definition of $z_{t}(\sigma)$.

Now, which reporting strategy does the agent use? Conditional on any $\sigma$, the agent receives the allocation $\left(c_{t}^{*}(\sigma), y_{t}^{*}(\sigma)\right)$. But because $\left(c^{*}, y^{*}\right)$ is incentive-compatible, it is at least weakly optimal for the agent to choose $\sigma^{*}$.

Because $\left(c^{*}, y^{*}, K^{*}\right)$ is feasible, the sequential markets clear.

Proposition 2 demonstrates that when we optimize over incentive-feasible allocations (as in Theorem 1), we are implicitly optimizing over capital-trading mechanisms. The following converse proposition shows that in any sequential markets equilibrium, the sign of the capital income taxes is the same as the sign of the wedge between intertemporal marginal rates of substitution and transformation.

Proposition 3. Let $U(c, l)=u(c)-v(l)$, where $u^{\prime},-u^{\prime \prime}>0$. Suppose $(c, k, r, K), k_{t}>0$ for all $t$, is a sequential markets equilibrium of a capital-trading mechanism $(z, y, \tau)$. Then:

$$
\left(1+\Phi_{K}\left(K_{t+1}, \int y_{t+1} d \mu\right)\left(1-\tau_{t+1}\right)-\delta\right)=u^{\prime}\left(c_{t}\right) / \beta E\left\{u^{\prime}\left(c_{t+1}\right) \mid F_{t}\right\} .
$$

Proof. Individual optimality and firm optimality imply that:

$$
\begin{aligned}
r_{t} & =\Phi_{K}\left(K_{t}, \int y_{t} d \mu\right) \\
u^{\prime}\left(c_{t}\right) & =\left(1+r_{t+1}\left(1-\tau_{t+1}\right)-\delta\right) \beta E\left\{u^{\prime}\left(c_{t+1}\right) \mid F_{t}\right\}
\end{aligned}
$$

which in turn implies the proposition.

Combining Propositions 2 and 3 with Corollary 1, we conclude that it is typically 
Pareto optimal for capital income taxes to be positive.

\section{Uniform Commodity Taxation}

In this section, we prove the uniform commodity taxation theorem. We return to the general setup described in the first section (with multiple commodities and a general production structure), except that we assume that utility is weakly separable:

$$
U(c, l)=V(u(c), l), u: R_{+}^{J} \rightarrow R_{+}
$$

We also assume that $u$ is strictly increasing and is continuously differentiable over the positive orthant of $R^{J}$. The notation $u_{j}$ and $G_{j}$ represents the partial derivatives of those functions with respect to their $j$ th arguments.

Theorem 2. Suppose $\Gamma^{*}\left(K_{1}\right)<\Gamma^{*}\left(K_{1}^{*}\right)$ for all $K_{1}<K_{1}^{*}$. Let $\left(c^{*}, y^{*}, K^{*}\right)$ solve $P 1\left(K_{1}^{*}\right)$ and suppose that there exists scalars $c^{+}, c_{+}$such that $c^{+}>c_{j t}^{*}\left(\theta^{T}\right)>c_{+}>0$ for all $j$ and for almost all $\theta^{T}$. Then:

$$
\begin{aligned}
& u_{j}\left(c_{t}^{*}\left(\theta^{T}\right)\right) / u_{k}\left(c_{t}^{*}\left(\theta^{T}\right)\right) \\
= & G_{j}\left(\int c_{t}^{*} d \mu, K_{t+1}^{*}, K_{t}^{*}, \int y_{t}^{*} d \mu\right) / G_{k}\left(\int c_{t}^{*} d \mu, K_{t+1}^{*}, K_{t}^{*}, \int y_{t}^{*} d \mu\right)
\end{aligned}
$$

for all $j, k$ and almost all $\theta^{T}$.

Proof. We claim that $c^{*}$ solves the following optimization problem MIN2:

$$
\min _{c} G\left(\int c_{t} d \mu, K_{t+1}^{*}, K_{t}^{*}, \int y_{t}^{*} d \mu\right)
$$




$$
\begin{aligned}
& \text { s.t. } u\left(c_{t}\right)=u\left(c_{t}^{*}\right) \text { a.e. } \\
& \text { s.t. } c_{t} \in L^{\infty}\left(F_{t}\right) \\
& \text { s.t. } c_{t} \geq 0 \text { a.e. }
\end{aligned}
$$

Suppose not. Then, there exists a nonnegative $c_{t}^{\prime}$ in $L^{\infty}\left(F_{t}\right)$ such that:

$$
G\left(\int c_{t}^{\prime} d \mu, K_{t+1}^{*}, K_{t}^{*}, \int y_{t}^{*} d \mu\right)<0
$$

and $u\left(c_{t}^{\prime}\left(\theta^{T}\right)\right)=u\left(c_{t}^{*}\left(\theta^{T}\right)\right)$ for all $\theta^{T}$ in $A \subseteq \Theta^{T}$, where $\mu(A)=1$. Let $c_{t}^{\prime \prime}\left(\theta^{T}\right)=c_{t}^{\prime}\left(\theta^{T}\right)$ for all $\theta^{T}$ in $A$ and $c_{t}^{\prime \prime}\left(\theta^{T}\right)=c_{t}^{*}\left(\theta^{T}\right)$ for all $\theta^{T}$ not in $A$. Let $c^{\prime \prime}=\left(c_{t}^{\prime \prime}, c_{-t}^{*}\right)$.

Clearly, $\left(c^{\prime \prime}, y^{*}, K^{*}\right)$ is feasible. It is also incentive-compatible because:

$$
\begin{aligned}
& W\left(\sigma^{*} ; c^{\prime \prime}, y^{*}\right) \\
= & W\left(\sigma^{*} ; c^{*}, y^{*}\right) \\
\geq & \max _{\sigma \in \Sigma} W\left(\sigma ; c^{*}, y^{*}\right) \\
= & \max _{\sigma \in \Sigma} W\left(\sigma ; c^{\prime \prime}, y^{*}\right)
\end{aligned}
$$

Thus, $\left(c^{\prime \prime}, y^{*}, K^{*}\right)$ also solves $P 1\left(K_{1}^{*}\right)$. However, because $G$ is strictly increasing in $K_{t+1}$, and strictly decreasing in $K_{t}$, there exists $K^{\prime}$ such that $\left(c^{\prime \prime}, y^{*}, K^{\prime}\right)$ solves $P 1\left(K_{1}\right)$ for some $K_{1}<K_{1}^{*}$. But this means that $\Gamma^{*}\left(K_{1}\right)=\Gamma^{*}\left(K_{1}^{*}\right)$ which is a contradiction.

Thus, $c^{*}$ solves the above minimization problem. The rest of the proof is simply technical: establishing that the solution to the minimization problem satisfies the first-order conditions in the theorem. We defer this to the Appendix. 
Thus, in a Pareto optimum, the marginal rate of substitution between two consumption goods is equalized to the marginal rate of transformation between those two goods. Because consumption only enters the incentive constraints through the sub-utility $u(c)$, the planner wants to find the way to deliver this sub-utility from consumption in a way that minimizes the resource cost of doing so.

Theorem 2 establishes a result about marginal rates of substitution and transformation. However, we can follow the line of attack in Section 3B to translate it into a statement about taxes. In particular, suppose agents can trade consumption goods in a competitive spot market in each period. Then, Theorem 2 implies that it is suboptimal for them to face taxes or subsidies in those markets that differ across consumption goods.

\section{Related Literature}

In this section, we discuss the connections between our paper and others in the literature. It is useful in doing so to divide the prior literature into two strands. The first analyzes model economies in which agents' private information affects their willingness to substitute between consumption goods (over time or within a period). Our paper is not in this literature. The second instead requires that agents' private information is independent of their marginal rates of substitution between consumption goods. Our paper provides generalizations of the prior results in this second literature.

In terms of the first strand of literature, there are now many papers on efficient dynamic insurance in the presence of hidden idiosyncratic shocks to endowments or tastes (see, among others, Townsend (1982), Green (1987), Thomas and Worrall (1990), Atkeson and Lucas (1992), Khan and Ravikumar (1997)). A key result that runs through this literature is that 
in Pareto optimal allocations, the typical agent's shadow interest rate exceeds the societal shadow interest rate. This result is similar to our Corollary 1. However, the result from the dynamic insurance literature depends crucially on the nature of the shock process to endowments or tastes.

To see this point, consider a two-period economy with a continuum of agents. The typical agent's endowment is $\left((1+\theta),(1+\theta)^{2}\right)$, where $\theta$ is random with positive support; the endowments are private information. Then, in an optimal allocation, agents' shadow interest rates are lower than the societal shadow interest rate. Intuitively, with hidden endowments, the direction of the gap depends on whether the agents who need insurance payments are more or less willing to substitute current for future consumption.

The other related papers all belong to the second strand of literature. Diamond and Mirrlees $(1978,1986)$ consider a special case of our general setup. In their model, agents are long-lived and can be disabled or not. Disabled agents are unproductive; able agents have known productivities. Once disabled, the agent stays disabled; the probability of an able agent becoming disabled is exogenous. The informational problem is that the disability status of the agent is known only to the agent. Diamond and Mirrlees prove that in the social optimum, the shadow societal interest rate is less than the private shadow interest rate. They argue explicitly that this result implies that capital income taxation is socially optimal. Our contribution over their work is that we generalize their positive capital income taxation result to a much larger class of individual productivity processes.

There are several papers on the properties of efficient allocations in the presence of repeated moral hazard (see, among others, Rogerson (1985), Phelan and Townsend (1991), Phelan (1994)). Again, in these settings the optimal allocations have the property that agents' 
shadow interest rates are higher than the societal shadow interest rate. The intuition behind this result is essentially the same as that behind Corollary 1. However, in this literature, the idiosyncratic output shocks are restricted to be independently and identically distributed; we instead allow for a much wider range of productivity shock processes.

We were originally motivated to write this paper by the work of da Costa and Werning (2001). They examine optimal monetary policy in two models (a cash-credit good framework and a shopping-time setup) in which agents are privately informed about their fixed productivities. In the cash-credit good framework, da Costa and Werning prove that if preferences is weakly separable between consumption and leisure, then the Friedman Rule (zero nominal interest rates) is socially optimal. This is essentially an implication of the uniform commodity taxation theorem, and so we conjecture that this result could be established in our more general setup. They also consider how deviations from weak separability of preferences affect optimal monetary policy.

In a paper written at the same time as ours, but independently, Werning (2001) analyzes the properties of optimal capital income taxes in a model economy with unobservable and heterogeneous fixed productivities. Like us (Corollary 2), he finds that it is optimal for capital income taxes to be zero in this setting.

\section{Conclusion}

In this paper, we consider the problem of optimal taxation when individual skills are unobservable, evolve stochastically over time, and are multi-dimensional. We show that when utility is weakly separable between consumption and leisure, it is optimal to equate the marginal rate of substitution between consumption goods for any agent to the marginal 
rate of transformation between those goods. It follows that Pareto optimal allocations are consistent with uniform taxation of all consumption goods.

We consider the intertemporal structure of optimal taxation when there is only a single consumption good and utility is additively separable between consumption and leisure. In this case, if the optimal allocation requires future consumption to be random given current information, then individuals face distorted consumption paths. We show that these distortions are consistent with the presence of positive capital income taxes.

Given additive separability of preferences between consumption and labor, the uniform commodity taxation theorem is generally valid, but the zero capital income taxation theorem is generally not. The reason for this distinction is that over time, individuals are acquiring information about their types. It is this idiosyncratic uncertainty that generates positive capital income taxes. In particular, if individuals knew their entire sequence of productivities in period 1, then we could use exactly the same reasoning as in Theorem 2 (or Corollary 2) to conclude that Pareto optimal allocations are consistent with zero capital income taxation.

We are able to prove the theorems in a highly general setting. We allow for a multidimensional specification of productivity. Individual productivities are independent over a continuum of individuals but follow arbitrary stochastic processes over time. As far as we are aware, these are the first characterizations of optimal tax policy for this kind of environment, with dynamically evolving private information and/or multiple-dimensional types.

The paper abstracts from government purchases. This is merely for notational convenience. The results can be easily extended to two kinds of model economies with government purchases. The first is one in which per-capita government purchases are a deterministic stream that the government must fund using taxes. The second is one in which government 
purchases are a choice variable for the social planner. In both kinds of models, the results are all valid regardless of how government purchases affect production or enter preferences. 


\section{Appendix}

In this appendix, we provide proofs of Theorems 1 and 2 for arbitrary Borel sets $\Theta$. We use the following notation:

$$
\begin{aligned}
L^{1}\left(F_{t}\right) & =\left\{x F_{t^{-}} \text {measurable }\left|\int\right| x \mid d \mu<\infty\right\} \\
L^{\infty}\left(F_{t}\right) & =\left\{x F_{t} \text {-measurable } \mid \exists A \in F_{t} \text { such that } \sup _{\theta^{T} \in A}|x|<\infty, \text { and } \mu(A)=1\right\}
\end{aligned}
$$

Let $\|.\|_{\infty}$ denote the usual norm on $L^{\infty}\left(F_{t}\right)$.

The general proofs of Theorems 1 and 2 are based on two results. The first is Theorem 1, p. 243 of Luenberger (1969). This theorem assumes that in an optimization problem with equality constraints, the objective and constraints are continuously Frechet differentiable in the neighborhood of a local optimum. It then proves that this local optimum must satisfy analogs of the usual Lagrangian first-order conditions.

The second key result is the following lemma. It establishes that as long as $c_{t}^{*}$ is bounded from above and below, the constraints in the minimization problems in the proofs of Theorems 1 and 2 are defined by a function that is continuously Frechet differentiable in a neighborhood of $c_{t}^{*}$.

Lemma 2. Let $u: R_{+} \rightarrow R$ and let $c_{t}^{*}$ be an element of $L^{\infty}\left(F_{t}\right)$. Suppose there exists scalars $c^{+}$and $c_{+}$such that $c^{+} \geq c_{t}^{*} \geq c_{+}>0$. Define $U: L^{\infty}\left(F_{t}\right) \rightarrow L^{1}\left(F_{t}\right)$ by:

$$
U\left(c_{t}\right)\left(\theta^{T}\right)=u\left(c_{t}\left(\theta^{T}\right)\right)
$$

Then $U$ is continuously Frechet differentiable in a neighborhood of $c_{t}^{*}$. 
Proof. Let $\eta_{t}$ be some element of $L^{\infty}\left(F_{t}\right)$ so that $\left|\eta_{t}\right|<c_{+} / 2$ a.e.. Let $\left\{\Delta_{n t}\right\}_{n=1}^{\infty}$ be an arbitrary sequence in $L^{\infty}\left(F_{t}\right)$ that converges to zero in the $L^{\infty}$ norm. Then, we can show that:

$$
\begin{aligned}
& \lim _{n \rightarrow \infty} \sup \int\left|u\left(c_{t}^{*}+\Delta_{n t}+\eta_{t}\right)-u\left(c_{t}^{*}+\eta_{t}\right)-u^{\prime}\left(c_{t}^{*}+\eta_{t}\right) \Delta_{n t}\right| d \mu /\left\|\Delta_{n t} \mid\right\|_{\infty} \\
= & \lim _{n \rightarrow \infty} \sup \int\left|\left(u\left(c_{t}^{*}+\Delta_{n t}+\eta_{t}\right)-u\left(c_{t}^{*}+\eta_{t}\right)\right) / \Delta_{n t}-u^{\prime}\left(c_{t}^{*}+\eta_{t}\right)\right|\left|\Delta_{n t}\right| d \mu /\left\|\Delta_{n t}\right\|_{\infty} \\
\leq & \lim _{n \rightarrow \infty} \sup \int\left|\left(u\left(c_{t}^{*}+\Delta_{n t}+\eta_{t}\right)-u\left(c_{t}^{*}+\eta_{t}\right)\right) / \Delta_{n t}-u^{\prime}\left(c_{t}^{*}+\eta_{t}\right)\right|\left(\left\|\Delta_{n t} \mid\right\|_{\infty}\right) d \mu /\left\|\Delta_{n t}\right\|_{\infty} \\
= & \lim _{n \rightarrow \infty} \sup \int\left|\left(u\left(c_{t}^{*}+\Delta_{n t}+\eta_{t}\right)-u\left(c_{t}^{*}+\eta_{t}\right)\right) / \Delta_{n t}-u^{\prime}\left(c_{t}^{*}+\eta_{t}\right)\right| d \mu \\
= & \lim _{n \rightarrow \infty} \sup \int\left|u^{\prime}\left(c_{t}^{*}+\lambda_{n} \Delta_{n t}+\eta_{t}\right)-u^{\prime}\left(c_{t}^{*}+\eta_{t}\right)\right| d \mu, 0<\lambda_{n}<1 \\
= & 0
\end{aligned}
$$

The last statement follows from the Bounded Convergence Theorem. In particular, $\mid u^{\prime}\left(c_{t}^{*}+\right.$ $\left.\lambda_{n} \Delta_{n t}+\eta_{t}\right)-u^{\prime}\left(c_{t}^{*}+\eta_{t}\right) \mid$ is bounded from above and converges to zero a.e. from the continuity of $u^{\prime}$.

It follows that in a neighborhood of $c_{t}^{*}$, the Frechet derivative of $U$ is well-defined and given by $U^{\prime}\left(c_{t}\right)(\Delta)=u^{\prime}\left(c_{t}\right) \Delta$ for all $\Delta$ in $L^{\infty}\left(F_{t}\right)$. The norm of this linear operator is given by

$\int\left|u^{\prime}\left(c_{t}\right)\right| d \mu$. But this is continuous in $c_{t}$ in a neighborhood of $c_{t}^{*}$. So $U$ is continuously Frechet differentiable in a neighborhood of $c_{t}^{*}$.

We can now turn to completing the proofs of Theorems 1 and 2.

\section{A1. Proof of Theorem 1}

Suppose we enlarge the constraint set by dropping the non-negativity constraints. The non-negative orthant of $L^{\infty}\left(F_{t}\right)$ has a non-empty interior. Hence, 0 must also be a local 
minimum of the enlarged minimization problem without the non-negativity constraints.

From Lemma 2 and Luenberger (1969; Theorem 1, page 243), we can conclude that there exists $z_{t+1}^{*} \in L^{\infty}\left(F_{t+1}\right)$ (the dual of $L^{1}\left(F_{t+1}\right)$ ) and $\lambda_{t}^{*} \in R$ such that 0 is a stationary point of the following Lagrangian.

$$
\begin{aligned}
& L\left(\zeta_{t}, \eta_{t}, \varepsilon_{t+1}\right) \\
= & \zeta_{t}+\int \eta_{t} d \mu+\lambda_{t}^{*}\left[\int \varepsilon_{t+1} d \mu-(1-\delta) \zeta_{t}-\Phi\left(K_{t+1}^{*}+\zeta_{t+1}, Y_{t+1}^{*}\right)\right] \\
& -\int z_{t+1}^{*}\left[u\left(c_{t}^{*}+\eta_{t}\right)+\beta u\left(c_{t+1}^{*}+\varepsilon_{t+1}\right)\right] d \mu
\end{aligned}
$$

In other words:

$$
\begin{aligned}
& 1-\lambda_{t}^{*}(1-\delta)-\Phi_{K}\left(K_{t+1}^{*}, Y_{t+1}^{*}\right) \lambda_{t}^{*}=0 \\
& \int \eta_{t} d \mu-\int z_{t+1}^{*} u^{\prime}\left(c_{t}^{*}\right) \eta_{t} d \mu=0 \text { for all } \eta_{t} \text { in } L^{\infty}\left(F_{t}\right) \\
& \lambda_{t}^{*} \int \varepsilon_{t+1} d \mu-\int z_{t+1}^{*} \beta u^{\prime}\left(c_{t+1}^{*}\right) \varepsilon_{t+1} d \mu=0 \text { for all } \varepsilon_{t+1} \text { in } L^{\infty}\left(F_{t+1}\right)
\end{aligned}
$$

Recall that $y=E\left\{x \mid F_{t}\right\}$ if $y$ is $F_{t}$-measurable and $\int x 1_{A} d \mu=\int y 1_{A} d \mu$ for all $A$ in $F_{t}$. It follows that:

$$
\begin{aligned}
\lambda_{t}^{*} & =\beta u^{\prime}\left(c_{t+1}^{*}\right) z_{t+1}^{*} \\
1 & =E\left\{u^{\prime}\left(c_{t}^{*}\right) z_{t+1}^{*} \mid F_{t}\right\} \\
\lambda_{t}^{*} & =\left[1-\delta+\Phi_{K}\left(K_{t+1}^{*}, Y_{t+1}^{*}\right)\right]^{-1}
\end{aligned}
$$

Theorem 1 follows. 


\section{A2. Proof of Theorem 2:}

Note that Lemma 2 can easily be extended to the case in which $c_{t}^{*}$ is a finite-dimensional random vector. As in the proof of Theorem 1, if we drop the non-negativity constraints from the minimization problem, we know that $c_{t}^{*}$ is a local minimum in the resulting problem. From Lemma 2, and Luenberger (1969; Theorem 1, p. 243), we know that there exists $z_{t}^{*} \in L^{\infty}\left(F_{t}\right)$ such that $c_{t}^{*}$ is a stationary point of the Lagrangian:

$$
L\left(c_{t}\right)=G\left(\int c_{t} d \mu, K_{t+1}^{*}, K_{t}^{*}, Y_{t}^{*}\right)-\int z_{t}^{*} u\left(c_{t}\right) d \mu
$$

In other words:

$$
\begin{aligned}
0 & =G_{j}\left(\int c_{t}^{*} d \mu, K_{t+1}^{*}, K_{t}^{*}, Y_{t}^{*}\right)-z_{t}^{*} u_{j}\left(c_{t}^{*}\right) \\
0 & =G_{k}\left(\int c_{t}^{*} d \mu, K_{t+1}^{*}, K_{t}^{*}, Y_{t}^{*}\right)-z_{t}^{*} u_{k}\left(c_{t}^{*}\right)
\end{aligned}
$$

Note that $z_{t}$ is positive because $G_{j}$ is positive. Then, we get the theorem. 


\section{References}

[1] Aiyagari, S. R., 1995, Optimal capital income taxation with incomplete markets, borrowing constraints, and constant discounting, Journal of Political Economy 103, 1158-1175.

[2] Atkinson, A., and Stiglitz, J.E., 1976, The design of tax structure: Direct versus indirect taxation, Journal of Public Economics 6, 55-75.

[3] Atkinson, A., and Stiglitz, J.E., 1980, Lectures on public economics, New York: McGrawHill.

[4] Chamley, C., 1986, Optimal taxation of capital income in general equilibrium with infinite lives, Econometrica 54, 607-622.

[5] Chari, V. V., and Kehoe, P., 1999, Optimal fiscal and monetary policy, in Handbook of Macroeconomics, ed. Taylor, J., and Woodford, M., New York: Elsevier.

[6] da Costa, C., and Werning, I., 2001, On the optimality of the Friedman Rule with heterogeneous agents and non-linear income taxation, University of Chicago manuscript.

[7] Diamond, P., and Mirrlees, J. A., 1978, A model of social insurance with variable retirement, Journal of Public Economics 10, 295-336.

[8] Diamond, P., and Mirrlees, J. A., 1986, Payroll-tax financed social insurance with variable retirement, Scandinavian Journal of Economics, 25-50.

[9] Garriga, C., 2001, Why are capital taxes high?, Universitat de Barcelona working paper. 
[10] Green, E., 1987, Lending and the smoothing of uninsurable income, in Contractual Arrangements for Intertemporal Trade, ed. E. Prescott and N. Wallace, Minneapolis: University of Minnesota Press, 3-25.

[11] Judd, K., 1985, Redistributive taxation in a simple perfect foresight model, Journal of Public Economics 28, 59-83.

[12] Khan, A., and Ravikumar, B., 2001, Growth and risksharing with private information, forthcoming, Journal of Monetary Economics.

[13] Luenberger, D., 1969, Optimization by vector space methods, New York: John Wiley and Sons.

[14] Mirrlees, J., 1971, An exploration in the theory of optimum income taxation, Review of Economic Studies 38, 175-208.

[15] Mirrlees, J., 1976, Optimal tax theory: A synthesis, Journal of Public Economics 6, $327-58$.

[16] Phelan, C., and Townsend, R., 1991, Comparing multi-period information constrained optima, Review of Economic Studies 58, 853-881.

[17] Phelan, C., 1994, Incentives and aggregate shocks, Review of Economic Studies 61, 681700.

[18] Rogerson, W., 1985, Repeated moral hazard, Econometrica 53, 69-76. 
[19] Storesletten, K., Telmer, C., and Yaron, A., 2001, How important are idiosyncratic shocks? Evidence from labor supply, American Economic Review Papers and Proceedings $91,413-41 \%$

[20] Thomas, J., and Worrall, T., 1990, Income fluctuation and asymmetric information: An example of a repeated principal-agent problem, Journal of Economic Theory 51, 367-90.

[21] Werning, I., 2001, Optimal dynamic taxation, University of Chicago manuscript. 